\title{
Efficient Compressed Sensing MR Image Reconstruction Using Anisotropic Overlapping Group Sparsity Total Variation
}

\author{
Linna $\mathrm{Wu}^{1}$, Yingpin Chen ${ }^{2}$ and Hongwei Du ${ }^{1+}$ \\ ${ }^{1}$ Centers for Biomedical Engineering, University of Science and Technology of China \\ ${ }^{2}$ School of Optoelectronic Information, University of Electronic science and Technology of China
}

\begin{abstract}
Compressed sensing magnetic resonance imaging has been proved to be an efficient method to reconstruct MR images from highly under-sampled $\mathrm{k}$-space data. Total variation (TV) and wavelet transform are two main sparsity expressions used as prior information in image recovery. In traditional TV, it just considers the sparse characteristic and ignores the group sparsity feature. In this paper, an extension of TV, named anisotropic overlapping sparse total variation (AOGSTV) is applied in the CS-MRI reconstruction process to reduce the staircase artifacts that always exist in TV model. A fast composite splitting algorithm (FCSA) is used to solve the AOGSTV problem. Radial sampling trajectory is used to under-sample the kspace data. Experimental results demonstrate that our proposed method can achieve better quality than the other state-of-the-art methods.
\end{abstract}

Keywords: Compressed Sensing, MRI, Anisotropic Overlapping Group Sparsity Total Variation, FCSA

\section{Introduction}

In compressed sensing magnetic resonance imaging (CS-MRI), total variation (TV) is always introduced as prior information in imaging reconstruction process [1]. Although TV has advantage in edge-preserving in MRI reconstruction, it just considers the sparsity feature and ignores other useful information, which leads to some undesired staircase artifacts [2]. To overcome the drawback mentioned above, some extensions of TV have been put forward. Total generalized variation (TGV) considers a certain order of differences instead of only first-order difference [3]. Nonlocal TV (NLTV) was studied for MRI reconstruction by considering the nonlocal information of an image [4]. Although TGV and NLTV have advantage in reducing staircase artifacts, they involve higher computational complexity than conventional TV method.

Recently, a novel denoising model named overlapping group sparsity total variation (OGSTV) was put forward for one-dimensional signal denoising [5]. It is assumed that the first-order difference of a signal is not only sparse but also contains a form of structured sparsity. That is, the large values of first-order difference function about a signal always arise near or adjacent to other large values, which can be regarded as a clustering or grouping property. The OGSTV model can reduce staircase artifacts efficiently and it can be solve by an majorization-minimization (MM) [6] algorithm that easy to calculate.

In this paper, we extend the OGSTV model to CS-MRI reconstruction to reduce the staircase artifacts that always appeared in standard TV model. There are two regularizers in the reconstruction formulation: OGSTV on the horizontal direction and OGSTV on the vertical direction of a MR image. We refer to this new combination as anisotropic OGSTV (AOGSTV). The fast composite splitting algorithm (FCSA) [7] is employed to solve this AOGSTV model by decomposing the composite regularization problem into two simpler sub-problems. A series of experiments are conducted to demonstrate the superiority of our method over other state-of-the-art methods in reconstructing images from highly under-sampled k-space data.

\section{Proposed method}

\footnotetext{
+ Corresponding author Tel.: (+86)13625510425;

E-mail address: duhw@ustc.edu.cn.
} 


\subsection{OGSTV for image denoising}

Given a signal $\mathrm{y} \in \mathrm{R}^{n \times 1}$ with white Gaussian noise $\sigma \in \mathrm{R}^{n \times 1}$, and $\mathrm{y}=\mathrm{x}+\sigma$. The unknown signal $\mathrm{x}$ can be solved by the following optimization problem:

$$
\mathrm{x}=\arg \min _{\mathrm{x}} \frac{1}{2}\|\mathrm{y}-\mathrm{x}\|_{2}^{2}+\lambda \phi(\mathrm{Dx})
$$

where D represents the first-order difference matrix and $\phi$ is a penalty function that promotes group sparsity. $\phi$ is defined as follows with group size $\mathrm{K}$ :

$$
\phi(\mathrm{v})=\sum_{\mathrm{n}}\left[\sum_{\mathrm{k}=0}^{\mathrm{K}-1}|\mathrm{v}(\mathrm{n}+\mathrm{k})|^{2}\right]^{1 / 2} .
$$

If $\mathrm{K}=1, \phi(\mathrm{v})$ is the standard TV. If $\mathrm{K}>1, \phi(\mathrm{v})$ is the OGSTV and (1) can be solved by MM algorithm.

\subsection{AOGSTV based CS-MRI}

When we consider OGSTV instead of TV as the penalty function in reconstructing process, the reconstruction model can be expressed in the following formulation:

$$
\mathrm{x}=\arg \min _{\mathrm{x}} \frac{1}{2}\|\mathrm{Ax}-\mathrm{y}\|_{2}^{2}+\alpha\|\mathrm{x}\|_{\text {AOGSTV }}
$$

where $\mathrm{x}$ is the vectorial form of an image to be reconstructed, $\mathrm{y}$ is the under-sampled $\mathrm{k}$-space data. We can extend the group sparsity concept [5] to a two-dimensional sparse signal $\mathrm{w} \in \mathrm{R}^{\mathrm{n} \times \mathrm{n}}$, in which it contains $\mathrm{K}$ square points that starting at index $(\mathrm{i}, \mathrm{j})$. Clearly,

$$
W_{i, j, K, K}=\left(\begin{array}{cccc}
v_{i, j} & v_{i, j+1} & \cdots & v_{i, j+K-1} \\
v_{i+1, j} & v_{i+1, j+1} & \cdots & v_{i+1, j+K-1} \\
\vdots & \vdots & \ddots & \vdots \\
v_{i+K-1, j} & v_{i+K-1, j+1} & \cdots & v_{i+K-1, j+K-1}
\end{array}\right) \in R^{K \times K} .
$$

The group size mentioned above is $\mathrm{K}^{2}$, it can be seen as a square block of $\mathrm{K} \times \mathrm{K}$ contiguous samples of w starting at index $(i, j)$. Here, we choose a group of entries adjacent to the objective point rather than a group following it along the horizontal and vertical direction only [8]. To our best knowledge, the former is better than latter in image reconstruction because there are more ambient pixels are considered. Then the overlapping group sparsity about two-dimensional signal can be defined as

$$
\varphi(\mathrm{w})=\sum_{\mathrm{i}=1}^{\mathrm{n}} \sum_{\mathrm{j}=1}^{\mathrm{n}}\left[\sum_{\mathrm{k} 1=0}^{\mathrm{K}-1} \sum_{\mathrm{k} 2=0}^{\mathrm{K}-1} \mathrm{w}_{\mathrm{i}+\mathrm{k} 1 \mathrm{j}+\mathrm{k} 2}^{2}\right]^{1 / 2} .
$$

When $i+k 1$ or $j+k 2$ outside the index of $w$, we take $w_{i+k 1, j+k 2}$ as zeros. The function mentioned above is convex. Next, mat means $\mathrm{R}^{\mathrm{n}^{2} \times 1} \rightarrow \mathrm{R}^{\mathrm{n} \times \mathrm{n}}$ and vec means $\mathrm{R}^{\mathrm{n} \times \mathrm{n}} \rightarrow \mathrm{R}^{\mathrm{n}^{2} \times 1}$. In this paper, we define the $\|\mathrm{x}\|_{\text {AOGSTV }}$ as follows:

$$
\|x\|_{\text {AOGSTV }}=\varphi\left(\operatorname{mat}\left(D_{\mathrm{h}} \mathrm{x}\right)\right)+\varphi\left(\operatorname{mat}\left(\mathrm{D}_{\mathrm{v}} \mathrm{x}\right)\right)
$$

where $D_{h}$ and $D_{v}$ are the horizontal and vertical direction first-order difference of an image, respectively.

\subsection{FCSA based MR image reconstruction method}

For the composite regularization problem (AOGSTV) in (3) and (6), we can solve it efficiently with FCSA algorithm by decomposing it into two simpler sub-problems as shown at below:

$$
\begin{aligned}
& \min _{x}\left\{1 / 2\|A x-y\|_{2}^{2}+\alpha \varphi\left(\operatorname{mat}\left(D_{h} x\right)\right)\right\}, \\
& \min _{x}\left\{1 / 2\|A x-y\|_{2}^{2}+\alpha \varphi\left(\operatorname{mat}\left(D_{v} x\right)\right)\right\} .
\end{aligned}
$$

In FISTA [9], authors consider the following optimization problem which can be extended into two parts:

$$
\min \{\mathrm{F}(\mathrm{x}) \equiv \mathrm{f}(\mathrm{x})+\gamma \mathrm{g}(\mathrm{x})\}, \mathrm{x} \in \mathrm{R}^{\mathrm{N}}
$$


where $f(x)=1 / 2\|A x-y\|_{2}^{2}$ is a smooth convex function which is continuously differential with Lipschitz constant $\mathrm{L}_{\mathrm{f}}$, and $\mathrm{g}(\mathrm{x})$ is a continuous non-smooth convex function like $\mathrm{L}_{1}$ norm, TV norm and $\operatorname{OGSTV}\left(\mathrm{L}_{21}\right)$ norm. Problem (9) can be reformulated as the following two sub-problems:

$$
\left\{\begin{array}{l}
\mathrm{y}^{\mathrm{k}+1}=\mathrm{x}^{\mathrm{k}}-\rho \nabla f\left(\mathrm{x}^{\mathrm{k}}\right) \\
\mathrm{x}^{\mathrm{k}+1}=\arg \min _{\mathrm{x}} \gamma \mathrm{g}(\mathrm{x})+\mathrm{L}_{\mathrm{f}} / 2\left\|\mathrm{x}-\mathrm{y}^{\mathrm{k}+1}\right\|_{2}^{2} .
\end{array}\right.
$$

When the sub-problem in (10) refers to OGSTV, the sub-problem (10) can be solved as follows:

$$
\mathrm{x}^{\mathrm{k}+1}=\operatorname{argmin}_{\mathrm{x}} \mathrm{L}_{\mathrm{f}}\left\|\mathrm{x}-\mathrm{y}^{\mathrm{k}+1}\right\|_{2}^{2}+\gamma \mathrm{x}^{\mathrm{T}} \mathrm{D}^{\mathrm{T}} \Lambda\left(\mathrm{Dx} \mathrm{x}^{\mathrm{k}}\right) \mathrm{Dx},
$$

where $[\Lambda(\mathrm{u})]_{\mathrm{m}, \mathrm{m}}=\sum_{\mathrm{i}=0}^{\mathrm{K}-1} \sum_{\mathrm{j}=0}^{\mathrm{K}-1}\left[\sum_{\mathrm{kl}=0}^{\mathrm{K}-1} \sum_{\mathrm{k} 2=0}^{\mathrm{K}-1}\left|\mathrm{u}_{\mathrm{m}-\mathrm{i}+\mathrm{k} 1, \mathrm{~m}-\mathrm{j}+\mathrm{k} 2}\right|^{2}\right]^{-1 / 2}$, D represents $\mathrm{D}_{\mathrm{h}}$ or $\mathrm{D}_{\mathrm{v}}$ and $\mathrm{u}=\operatorname{mat}(\mathrm{Dx})$. The entries of $\Lambda$ can be figured out by using conv2 function. By using MM algorithm, $\mathrm{x}^{\mathrm{k}+1}$ can be solved as:

$$
\mathrm{x}^{\mathrm{k}+1}=\mathrm{y}^{\mathrm{k}+1}-\mathrm{D}^{\mathrm{T}}\left(\frac{1}{\gamma} \Lambda^{-1}\left(\mathrm{Dx}{ }^{\mathrm{k}}\right)+\mathrm{DD}^{\mathrm{T}}\right)^{-1} \mathrm{Dy}^{\mathrm{k}+1} .
$$

Then the FCSA-based CS-MRI reconstruction is summarized in Algorithm 1.

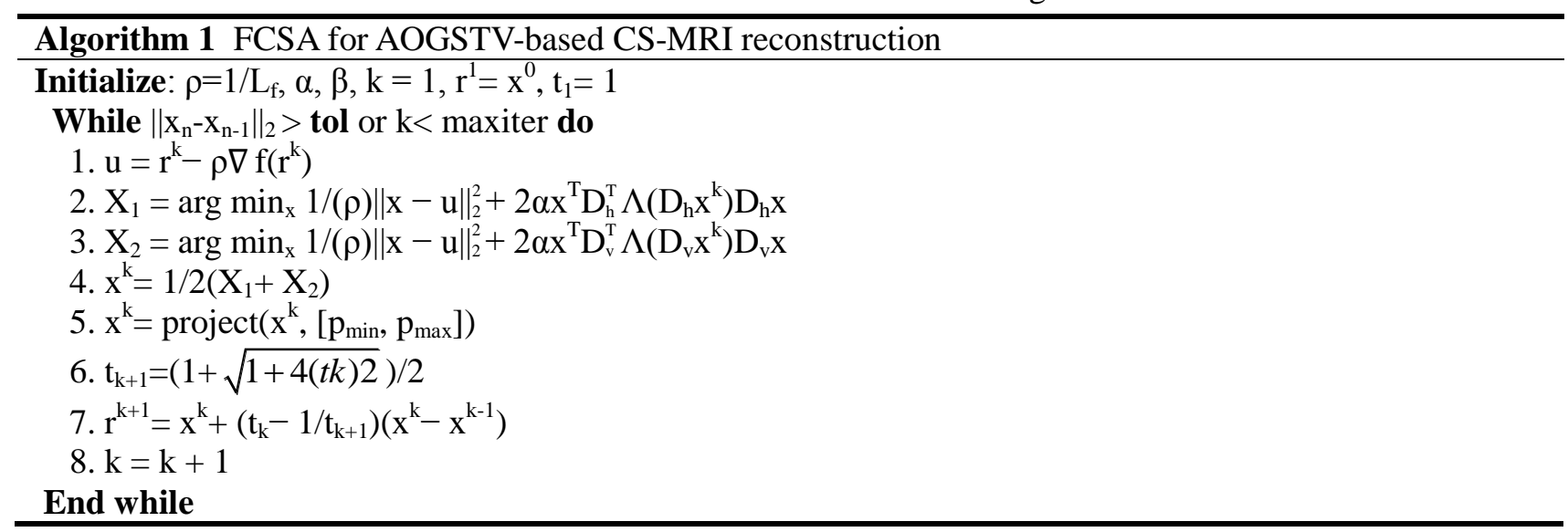

\section{Materials and methods}

In the experiments, neck MR raw data in Fig.1 (a) are employed to demonstrate the superiority of the proposed AOGSTV-based CS-MRI reconstruction method. The k-space data are obtained from a GE MR750 3T scanner. Radial sampling pattern in Fig. 1(b) is implemented to under-sample the k-space data.

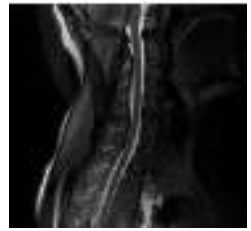

(a)

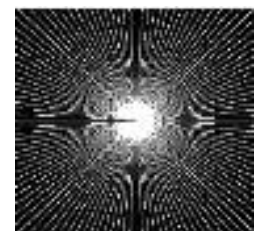

(b)

Fig. 1: The under sampling trajectory and original MR image: (a) Neck MR image. (b) Radial under-sample pattern.

In the following experiments, we compare our proposed method to the state-of-the-art algorithms such as CG [10], TVCMRI [11], RecPF [12], FCSA [6], WaTMRI [13] and FICOTA [14] at various sampling ratios. All the core codes are downloaded from the authors' websites. The simulation processes are carried out in the same MATLAB programming environment, i.e. version R2015a. The experiments are conducted on a desktop computer with $3.2 \mathrm{GHz}$ Inter Core processor, $4 \mathrm{G}$ memory and windows 7 operating system and the white Gaussian noise with 0.01 standard deviation is added to k-space data. The parameter $\alpha$ is set as 0.05 . We calculate Signal-to-Noise Ratio (SNR) and Structural Similarity (SSIM) [14] for comparison.

\section{Experiments and results}

\subsection{Study on group size $K$}


As shown in Fig.2, the group size $\mathrm{K}$ changes from 1 to 15 to test its influence on image reconstruction results. If $\mathrm{K}=1$, then $\varphi(\mathrm{w})=\|\mathrm{w}\|_{1}$ and $\|\mathrm{x}\|_{\text {AOGSTV }}$ is the standard anisotropic total variation (ATV). We can find that when the group size $\mathrm{K}$ changes from 1 to 2 , the reconstructed image quality (SNR and SSIM) arises great. The best group size for reconstruction is 3 . When $\mathrm{K}>3$, the image quality becomes decline as $\mathrm{K}$ increases.

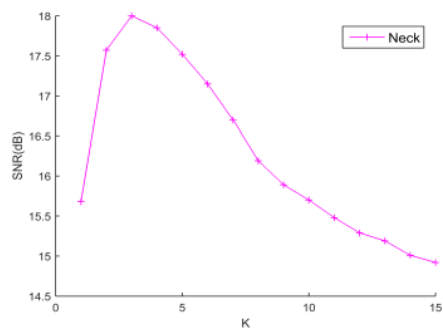

(a)

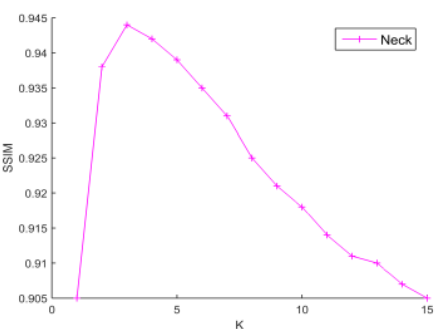

(b)

Fig. 2: The SNRs and SSIMs of different group size $\mathrm{K}$ from 1 to 15 by $24.85 \%$ radial under-sample ratio.

As the zoomed-in areas presented to us in Fig.3 (a) and Fig.3 (b), when K is 60, the reconstructed image becomes smoother and image resolution decreases. From Fig.2, we can also find that the values of SNR decline faster than SSIM. When K is 14 , the value of SNR is smaller than that when K is 1 , while SSIM is contrary.

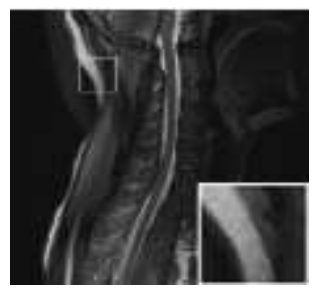

(a)

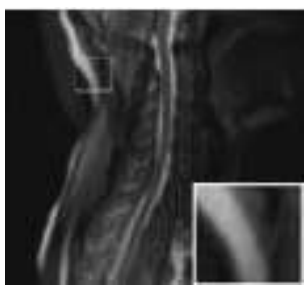

(b)

Fig. 3: The reconstructed and zoomed-in images, the sampling ratio is set as $24.85 \%$. (a) $K=3$; (b) $K=60$.

\subsection{Neck MR image experiments}

The neck MR image raw data are acquired under a T2-weighted Fast Recovery Fast Spin Echo (FRFSE) pulse sequence with the following parameters: Filed of View $(\mathrm{FOV})=24 \mathrm{~cm}, \mathrm{TR}=2500 \mathrm{~ms}, \mathrm{TE}=110 \mathrm{~ms}$ and matrix size $=256 \times 256$. The reconstruction results from different algorithms are displayed in Fig.4. The iterations are all set as 50. The curve graphs in Fig.4 show the superiority of the proposed AOGSTV method over the other methods in holding highest value of SNR and keeping image structural information (SSIM).

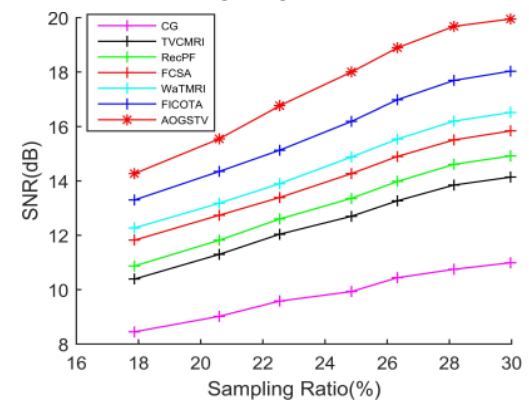

(a)

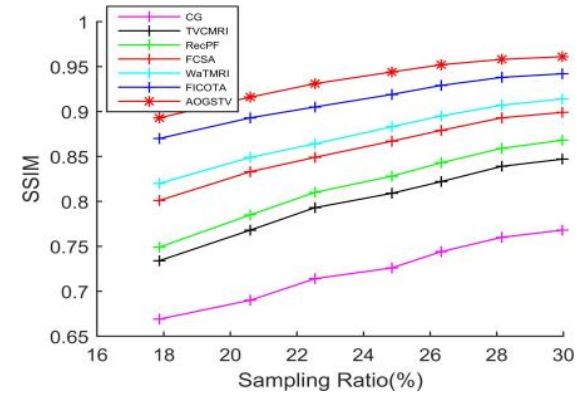

(b)

Fig. 4: SNRs and SSIMs under various sampling ratios for neck image reconstruction with different methods.

The visual results at the sampling ratio of $24.85 \%$ are shown in Fig.5. As the reconstructed images and related zoomed-in parts displayed, the proposed method outperforms the other methods prominently. The proposed AOGSTV method has absolutely advantage in reducing staircase artifacts.

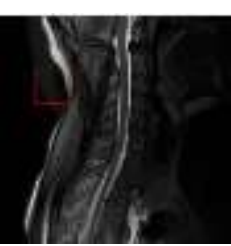

(a)

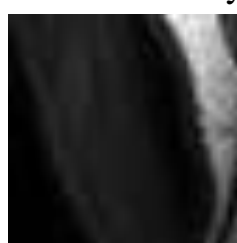

(b)

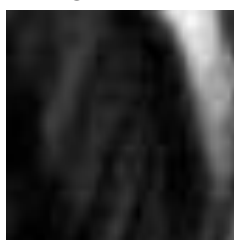

(c)

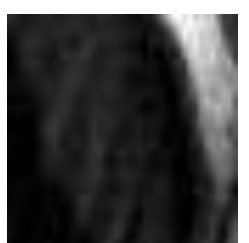

(d)

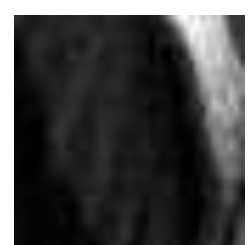

(e) 


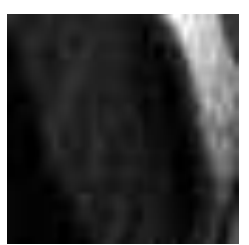

(f)

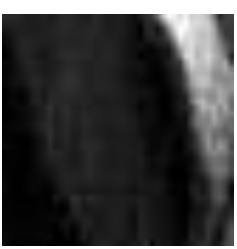

(g)

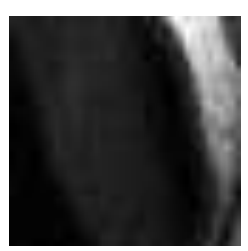

(h)

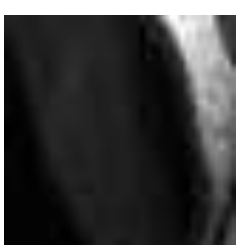

(i)

Fig. 5: Neck MR image and part zoomed-in reconstruction results by different methods. (a) original neck image; (b) part zoomed-in original image; (c) CG method; (d) TVCMRI method; (e) RecPF method; (f) FCSA method; (g) WaTMRI method; (h) FICOTA method; (i) AOGSTV method.

\section{Discussion}

The proposed AOGSTV method considers the overlapping group sparsity characteristic instead of only the sparsity feature of total variation. By taking account into the adjacent values of one objective point, AOGSTV method can alleviates the staircase artifacts that often appear in total variation effectively. When the group size $\mathrm{K}$ is larger than 3, the reconstructed image becomes smoother as $\mathrm{K}$ increases, and the image resolution declines. So in the CS-MRI reconstruction process, we should choose an appropriate $\mathrm{K}$ for the best image quality. How to apply the AOGSTV to parallel MR images and dynamic MR images is the next work we want to exploit.

\section{Conclusion}

In this paper, an AOGSTV-based method is put forward for CS-MRI reconstruction. When k-space data are highly under-sampled, it can get less staircase artifacts that caused by TV. We solve the AOGSTV problem by FCSA algorithm, and divide it into two simpler sub-problems which can be solved by FISTA. The experimental results indicate that the proposed method is capable of achieving significant improvement of image quality with highest value of SNR and the strongest ability to keep image structural information.

\section{Acknowledgements}

This work is supported by National Nature Science Foundation of China under Grant No.81527802.

\section{References}

[1] Lustig M, Donoho D L, Santos J M, et al. Compressed sensing MRI. IEEE signal processing magazine, 2008, 25(2): 72-82.

[2] Chambolle A, Lions P L. Image recovery via total variation minimization and related problems. Numerische Mathematik, 1997, 76(2): 167-188.

[3] Knoll F, Bredies K, Pock T, et al. Second order total generalized variation (TGV) for MRI. Magnetic resonance in medicine, 2011, 65(2): 480-491.

[4] Huang J, Yang F. Compressed magnetic resonance imaging based on wavelet sparsity and nonlocal total variation//Biomedical Imaging (ISBI), 2012 9th IEEE International Symposium on. IEEE, 2012: 968-971.

[5] Selesnick I W, Chen P Y. Total variation denoising with overlapping group sparsity//Acoustics, Speech and Signal Processing (ICASSP), 2013 IEEE International Conference on. IEEE, 2013: 5696-5700.

[6] Figueiredo M A T, Bioucas-Dias J M, Nowak R D. Majorization-minimization algorithms for wavelet-based image restoration. IEEE Transactions on Image processing, 2007, 16(12): 2980-2991.

[7] Huang J, Zhang S, Li H, et al. Composite splitting algorithms for convex optimization. Computer Vision and Image Understanding, 2011, 115(12): 1610-1622.

[8] Chen P Y, Selesnick I W. Translation-invariant shrinkage/thresholding of group sparse signals. Signal Processing, 2014, 94: 476-489.

[9] Beck A, Teboulle M. A fast iterative shrinkage-thresholding algorithm for linear inverse problems[J]. SIAM journal on imaging sciences, 2009, 2(1): 183-202.

[10] Hong M, Yu Y, Wang H, et al. Compressed sensing MRI with singular value decomposition-based sparsity basis. Physics in medicine and biology, 2011, 56(19): 6311. 
[11] Ma S, Yin W, Zhang Y, et al. An efficient algorithm for compressed MR imaging using total variation and wavelets//Computer Vision and Pattern Recognition, 2008. CVPR 2008. IEEE Conference on. IEEE, 2008: 1-8.

[12] Yang J, Zhang Y, Yin W. A fast alternating direction method for TVL1-L2 signal reconstruction from partial Fourier data. IEEE Journal of Selected Topics in Signal Processing, 2010, 4(2): 288-297.

[13] Chen C, Huang J. Compressive sensing MRI with wavelet tree sparsity//Advances in neural information processing systems. 2012: 1115-1123.

[14] Hao W, Li J, Qu X, et al. Fast iterative contourlet thresholding for compressed sensing MRI. Electronics Letters, 2013, 49(19): 1206-1208.

[15] Wang Z, Bovik A C, Sheikh H R, et al. Image quality assessment: from error visibility to structural similarity. IEEE transactions on image processing, 2004, 13(4): 600-612. 